\title{
Coronagraph focal-plane phase masks based on photonic crystal technology: recent progress and observational strategy
}

\author{
Naoshi Murakami ${ }^{* a}$, Jun Nishikawa $^{\mathrm{b}}$, Wesley A. Traub ${ }^{\mathrm{c}}$, Dimitri Mawet ${ }^{\mathrm{c}, \mathrm{d}}$, \\ Dwight C. Moody ${ }^{c}$, Brian D. Kern ${ }^{c}$, John T. Trauger ${ }^{c}$, Eugene Serabyn ${ }^{c}$, \\ Shoki Hamaguchi ${ }^{\mathrm{e}}$, Fumika Oshiyama ${ }^{\mathrm{e}}$, Moritsugu Sakamoto ${ }^{\mathrm{e}}$, Akitoshi Ise ${ }^{* * \mathrm{e}}$, \\ Kazuhiko Oka ${ }^{\mathrm{a}}$, Naoshi Baba ${ }^{\mathrm{a}}$, Hiroshi Murakami ${ }^{\mathrm{f}}$, Motohide Tamura ${ }^{\mathrm{b}}$ \\ ${ }^{\mathrm{a}}$ Division of Applied Physics, Faculty of Engineering, Hokkaido University, Sapporo, Hokkaido \\ 060-8628, Japan; \\ ${ }^{\mathrm{b}}$ National Astronomical Observatory of Japan, 2-21-1 Osawa, Mitaka, Tokyo 181-8588, Japan; \\ ${ }^{\mathrm{c}}$ Jet Propulsion Laboratory, California Institute of Technology, 4800 Oak Grove Dr, Pasadena, CA \\ 91109, USA; \\ ${ }^{\mathrm{d}}$ European Southern Observatory, Alonso de Cordova 3107, Vitacura, Santiago 763 0355, Chile; \\ ${ }^{\mathrm{e}}$ Division of Applied Physics, Graduate School of Engineering, Hokkaido University, Sapporo, \\ Hokkaido 060-8628, Japan; \\ ${ }^{\mathrm{f}}$ Institute of Space and Astronautical Science, Japan Aerospace Exploration Agency (ISAS/JAXA), \\ 3-1-1 Yoshinodai, Chuo-ku, Sagamihara 252-0222, Japan
}

\begin{abstract}
Photonic crystal, an artificial periodic nanostructure of refractive indices, is one of the attractive technologies for coronagraph focal-plane masks aiming at direct imaging and characterization of terrestrial extrasolar planets. We manufactured the eight-octant phase mask (8OPM) and the vector vortex coronagraph (VVC) mask very precisely using the photonic crystal technology. Fully achromatic phase-mask coronagraphs can be realized by applying appropriate polarization filters to the masks. We carried out laboratory experiments of the polarization-filtered 8OPM coronagraph using the High-Contrast Imaging Testbed (HCIT), a state-of-the-art coronagraph simulator at the Jet Propulsion Laboratory (JPL). We report the experimental results of $10^{-8}$-level contrast across several wavelengths over $10 \%$ bandwidth around $800 \mathrm{~nm}$. In addition, we present future prospects and observational strategy for the photonic-crystal mask coronagraphs combined with differential imaging techniques to reach higher contrast. We proposed to apply the polarization-differential imaging (PDI) technique to the VVC, in which we built a two-channel coronagraph using polarizing beam splitters to avoid a loss of intensity due to the polarization filters. We also proposed to apply the angular-differential imaging (ADI) technique to the 8OPM coronagraph. The 8OPM/ADI mode mitigates an intensity loss due to a phase transition of the mask and provides a full field of view around central stars. We present results of preliminary laboratory demonstrations of the PDI and ADI observational modes with the phase-mask coronagraphs.
\end{abstract}

Keywords: high-contrast imaging, extrasolar planets, photonic crystal, coronagraphy, eight-octant phase-mask coronagraph, vector vortex coronagraph, polarization

\section{INTRODUCTION}

Direct imaging and characterization of extrasolar planets is one of the most attractive issues in modern astronomy. Recently, giant planets with several Jupiter masses have been directly imaged ${ }^{1-3}$. However, direct imaging of Earth-like planets is extremely challenging because of overwhelming intensity ratios (contrasts), such as $10^{-10}$, between an Earthlike planet and its parent star. For solving this problem, high-contrast instruments are indispensable for strongly suppressing the bright starlight.

\footnotetext{
*nmurakami@eng.hokudai.ac.jp; phone 8111 706-6720; fax 8111 706-7811

** Current affiliation: Nikon Corporation
}

Space Telescopes and Instrumentation 2012: Optical, Infrared, and Millimeter Wave, edited by Mark C. Clampin, Giovanni G. Fazio, Howard A. MacEwen, Jacobus M. Oschmann, Jr., Proc. of SPIE Vol. 8442, 844205

(C) 2012 SPIE · CCC code: 0277-786/12/\$18 - doi: 10.1117/12.925613 
Focal-plane phase-mask coronagraphs are promising methods which theoretically realize perfect stellar elimination. Several kinds of coronagraph focal-plane masks have been proposed, for example, four-quadrant phase mask (4QPM) ${ }^{4}$, eight-octant phase mask (8OPM) ${ }^{5}$, optical or vector vortex coronagraph (OVC or VVC) masks ${ }^{6,7}$, and so on. The 4QPM and 8OPM divide a stellar image into four or eight sectors, and provide $\pi$ phase difference between lights passing through the adjacent sectors. The OVC (or VVC) mask provides a helical phase structure $l_{p} \times \theta$, as a function of an azimuth angle $\theta\left(l_{p}\right.$ is the so-called topological charge). It has been shown that $4 \mathrm{~N}$-segment phase masks or even-charged vortex masks can realize perfect stellar elimination ${ }^{7,8}$. However, it is difficult to manufacture the focal-plane phase masks, because the masks have phase singular point at the center, and also because an achromatic phase modulation is very challenging.

In this paper, we report our recent activities for developing the phase mask coronagraphs based on the photonic crystal technology. We manufactured the photonic-crystal 8OPM and VVC mask, which have extremely small central singular points. The masks are placed between polarization filters for realizing an achromatic phase modulation utilizing a Pancharatnam-Berry's phase ${ }^{9,10}$. We carried out laboratory demonstration of the 8OPM coronagraph using the JPL high contrast imaging testbed (HCIT). We also proposed to combine the phase mask coronagraphs with differential imaging techniques to enhance the achievable contrast. In the latter part of the paper, we report preliminary experimental results, acquired using a coronagraph simulator constructed at the laboratory in Hokkaido University, of the 8OPM coronagraph with an angular differential imaging (ADI) mode, and the VVC with a polarization-differential imaging (PDI) mode.

\section{PHOTONIC-CRYSTAL CORONAGRAPH MASKS}

The photonic crystal is an artificial periodic nanostructure of refractive indices. The photonic crystal is an attractive device for the coronagraph focal-plane phase masks because of its extremely small manufacturing defect.

Figure 1 shows a schematic cross-sectional view of the photonic-crystal coronagraph masks, manufactured by the Photonic Lattice Inc. A periodic corrugated structure is formed by means of electron-beam lithography on a substrate (synthetic fused silica) with a thickness of $1 \mathrm{~mm}$. Two dielectric materials, $\mathrm{Nb}_{2} \mathrm{O}_{5}$ and $\mathrm{SiO}_{2}$, are then deposited alternatively on the substrate by RF bias sputtering. The corrugated pattern formed on the substrate is preserved during the deposition of the two materials by the autocloning technique ${ }^{11,12}$. We note that a thickness of the multilayer (about 130 layers) on the substrate is about $5 \mu \mathrm{m}$. The manufactured masks exhibit birefringent characteristics, and become half-wave plates (HWP) at designed wavelengths with space-variant fast and slow axes along the corrugated patterns.

When a centrally-focused, right-handed circularly polarized light enters to the space-variant HWP, the output light is converted to left-handed circularly polarized light. Then, the output light has an additional phase according to $2 \alpha$ ( $\alpha$ is an orientation angle of the optical axis of the HWP). This additional phase is known as the Pancharatnam-Berry's phase ${ }^{9,10}$. Thus the space-variant HWP enables to embed the space-variant arbitrary phase on the incoming circularly polarized light. For the specific case of $\alpha= \pm 45^{\circ}$, the HWP coverts an incoming $0^{\circ}$ linearly polarized light to that with an angle of $90^{\circ}$, and embeds a $\pi$-phase difference between the lights passing through the HWP with $\alpha=45^{\circ}$ and $-45^{\circ}$. Thus the spacevariant HWP is applicable both for the VVC mask and the 8OPM.

Figure 2 shows a picture and a scanning electron microscope (SEM) image of the manufactured photonic-crystal 8OPM. The directions of the stripes on the surface $\left(\alpha= \pm 45^{\circ}\right)$ correspond to the fast axes of the space-variant HWP. As described above, this mask provides the $\pi$-phase difference between light beams passing through the adjacent octant regions. From the SEM image, we can see that manufacturing defect is extremely small, which has been roughly estimated to be about $400 \mathrm{~nm}$ for a central singular point and about $100 \mathrm{~nm}$ for octant boundaries ${ }^{13}$.

In fig. 2, we also show a magnified image of the manufactured VVC mask seen between two crossed polarizers. The radial fast axes, shown by arrows, embed a $2 \times 2 \pi$ phase ramp on an incoming light. Thus this axially-symmetric HWP acts as an optical vortex mask with a topological charge of 2 .

In general, a retardation of a wave plate depends on a wavelength, and thus it is difficult to realize an achromatic wave plate over a broad spectral range ${ }^{7}$. For the focal-plane masks utilizing the Pancharatnam-Berry's phase, the coronagraph performance would severely degrade due to an error of the retardation. The polarization filtering will be a promising solution for broadening the operation spectral range of the coronagraph ${ }^{14}$. For realizing the achromatic coronagraphs, it has been proposed to use crossed linear polarizers for the $8 \mathrm{OPM}^{5,13}$, or circular polarizers for the VVC mask ${ }^{15}$. 


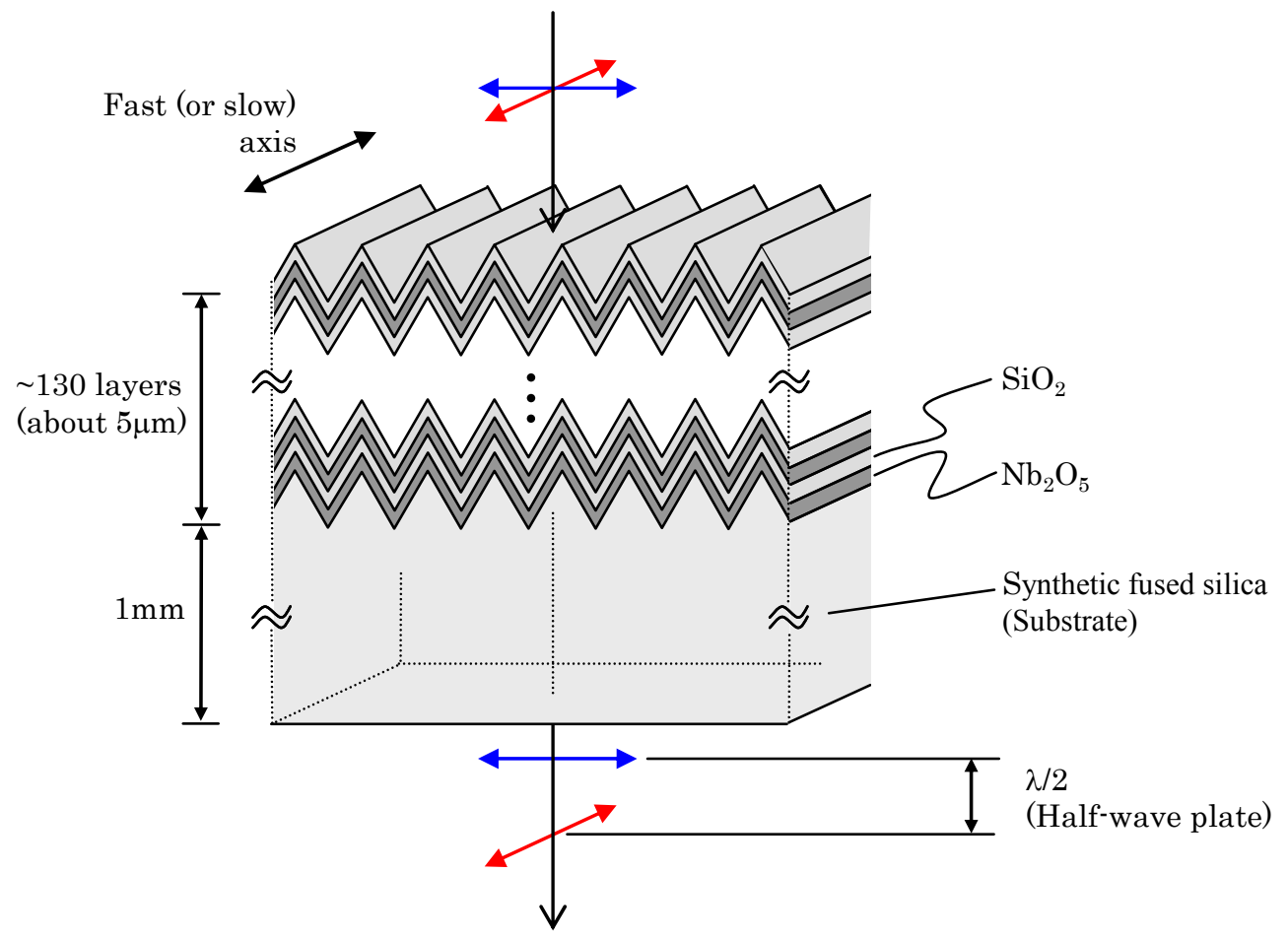

Figure 1. A schematic cross-sectional view of a photonic-crystal phase mask based on the autocloning technique.
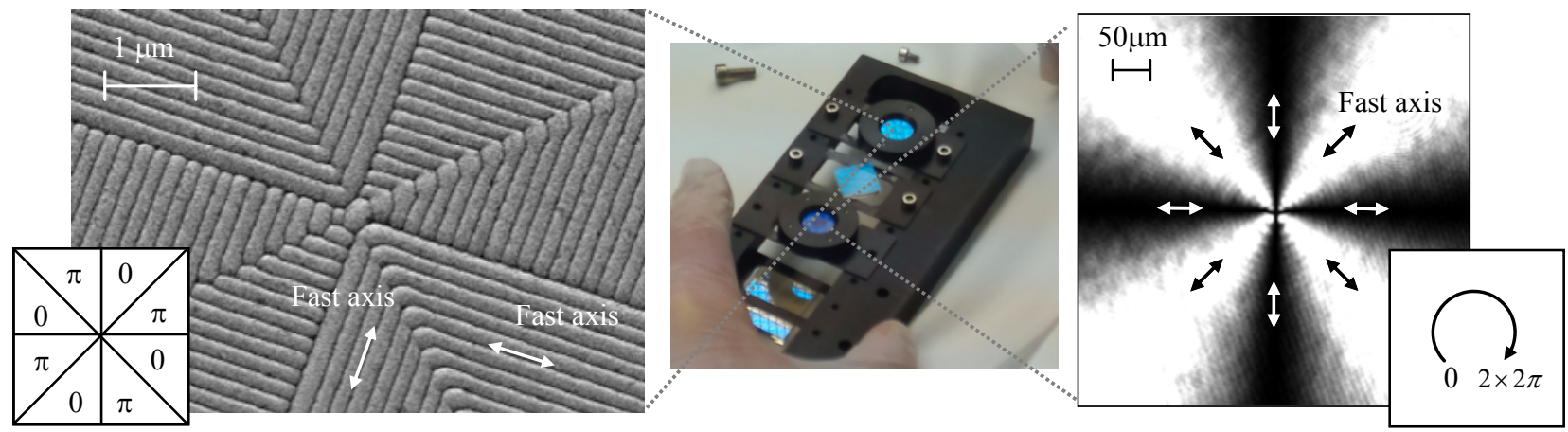

Figure 2. A picture and microscope images of an eight-octant phase mask (left) and a vector vortex coronagraph mask (right). The microscope image is taken by placing the VVC mask between two crossed polarizers.

\section{LABORATORY DEMONSTRATION AT HCIT/JPL}

\subsection{The High-Contrast Imaging Testbed (HCIT)}

We carried out laboratory demonstration of the 8OPM coronagraph on the high contrast imaging testbed (HCIT) at the Jet Propulsion Laboratory (JPL). The HCIT is a state-of-the-art coronagraph simulator installed into a vacuum chamber. Several kinds of coronagraphs have been tested at the HCIT/JPL ${ }^{16-22}$.

An optical configuration of the HCIT for the focal-plane coronagraph masks are shown in Fig. 2 of Ref. [22]. Several kinds of light sources are available, such as a monochromatic laser of $\lambda=785 \mathrm{~nm}$, or a supercontinuum light with selectable bandpass filters with bandwidths of $2 \%, 10 \%$, and $20 \%$. Light from a pinhole (a model star) is collimated by 
an off-axis parabolic (OAP) mirror, and reflected on a Xinetics $64 \times 64$ deformable mirror (DM). An image of the model star is formed on a focal plane by a second OAP with a focal length of about $1500 \mathrm{~mm}$. A polarizer (a diameter of $D=19.5 \mathrm{~mm}$ ) is placed between the DM and the second OAP for chromatic leakage filtering. The polarizer defines a size of an entrance pupil. Then, a physical scale of $\lambda / D$ becomes about $62 \mu \mathrm{m}$ on the focal plane where the photonic-crystal $8 \mathrm{OPM}$ is placed. Behind the $8 \mathrm{OPM}$, the entrance pupil is reimaged by a third OAP with a magnification of about 0.52 , and a Lyot stop (a diameter of $9 \mathrm{~mm}$ corresponding to $90 \%$ of the entrance pupil) is placed on the reimaged pupil. A second polarizer is placed behind the Lyot stop for the polarization filtering. The model star is imaged on a detector with a magnification of about 3 with respect to the first focal plane where the 8OPM is placed.

The DM is used to correct the wavefront error due to imperfection of the optical elements, and to create a "dark hole" against the residual speckle pattern of the model star. The algorithm for the wavefront correction is called the electric field conjugation (EFC), because the DM actuators are controlled so as to create the negative of the electric field of the speckle pattern ${ }^{23}$. The electric field created by the DM actuators is destructively interfered with the speckle pattern on the detector. After the DM control, the electric field of the speckle pattern is measured again, and then the wavefront is corrected iteratively to create a deeper dark hole.

A possible smallest inner working angle (IWA) is set to $2 \lambda / D$ by the $8 \mathrm{OPM}$ itself. A possible largest outer working angle (OWA), on the other hand, is defined by the number of the DM actuators across the light beam (about 19 actuators resulting in the possible OWA of about $9 \lambda / D$ ). In our experiments, we set the IWA and OWA for the EFC operation to $2.7 \lambda / D$ and $7 \lambda / D$, respectively. We note that the IWA is not limited by the coronagraph, but by the wavefront control system because of the difficulty of controlling low order aberrations.

\subsection{Results of the laboratory demonstrations}

In fig. 3 , we show a result of the 8 OPM coronagraph acquired using the supercontinuum light source with the $20 \%$ bandpass filter (a center wavelength of $800 \mathrm{~nm}$ and a bandwidth of $160 \mathrm{~nm}$ ). We note that an effective bandwidth is smaller than $20 \%$ depending on a shape of the spectrum of the light source. The right graph shows a contrast profile along a horizontal line passing through a center of the dark hole (a white dashed line shown in the coronagraph image). We can see that a high contrast of about $2.4 \times 10^{-8}$ can be achieved over the broad spectral range.
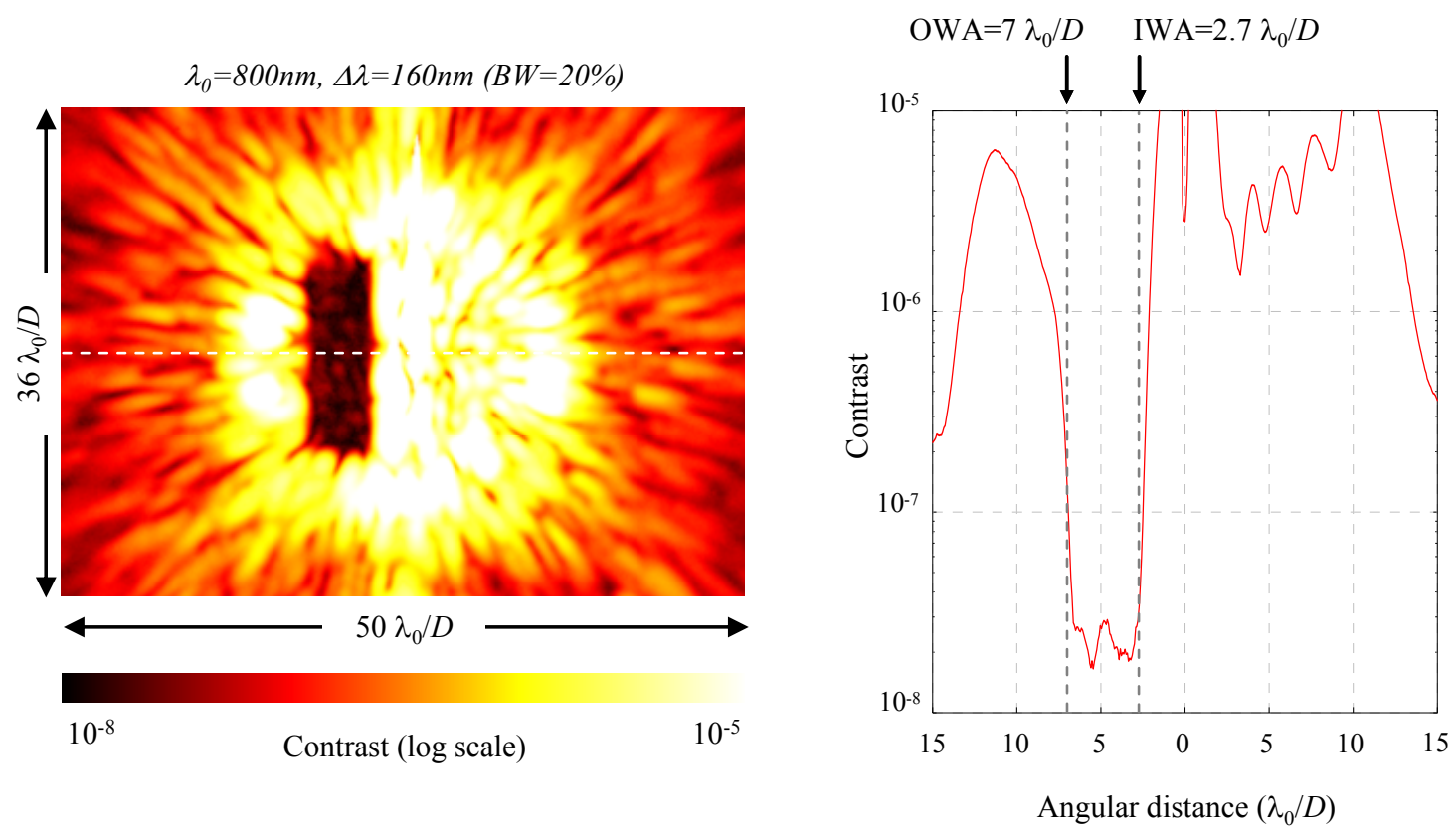

Figure 3. The 8 OPM coronagraph image acquired with a broadband light source with a $20 \%$ bandpass filter $(720-880 \mathrm{~nm})$. A dark hole is generated over a range between $2.7 \lambda / D$ and $7 \lambda / D$ from a suppressed model star. The graph at the right side shows an intensity profile along a central horizontal line shown by a white dashed line in the image. 
Figure 4 shows results of the 8 OPM coronagraph acquired with five $2 \%$ bandpass filters at five contiguous wavelengths around $800 \mathrm{~nm}$. Achieved contrasts over the dark holes are shown beneath the images and in the bottom graph. The best contrast could be obtained at $\lambda=800 \mathrm{~nm}$, because the wavefront control is driven only at this wavelength. An average contrast over the $10 \%$ bandwidth covered by the five wavelengths is about $2.1 \times 10^{-8}$.
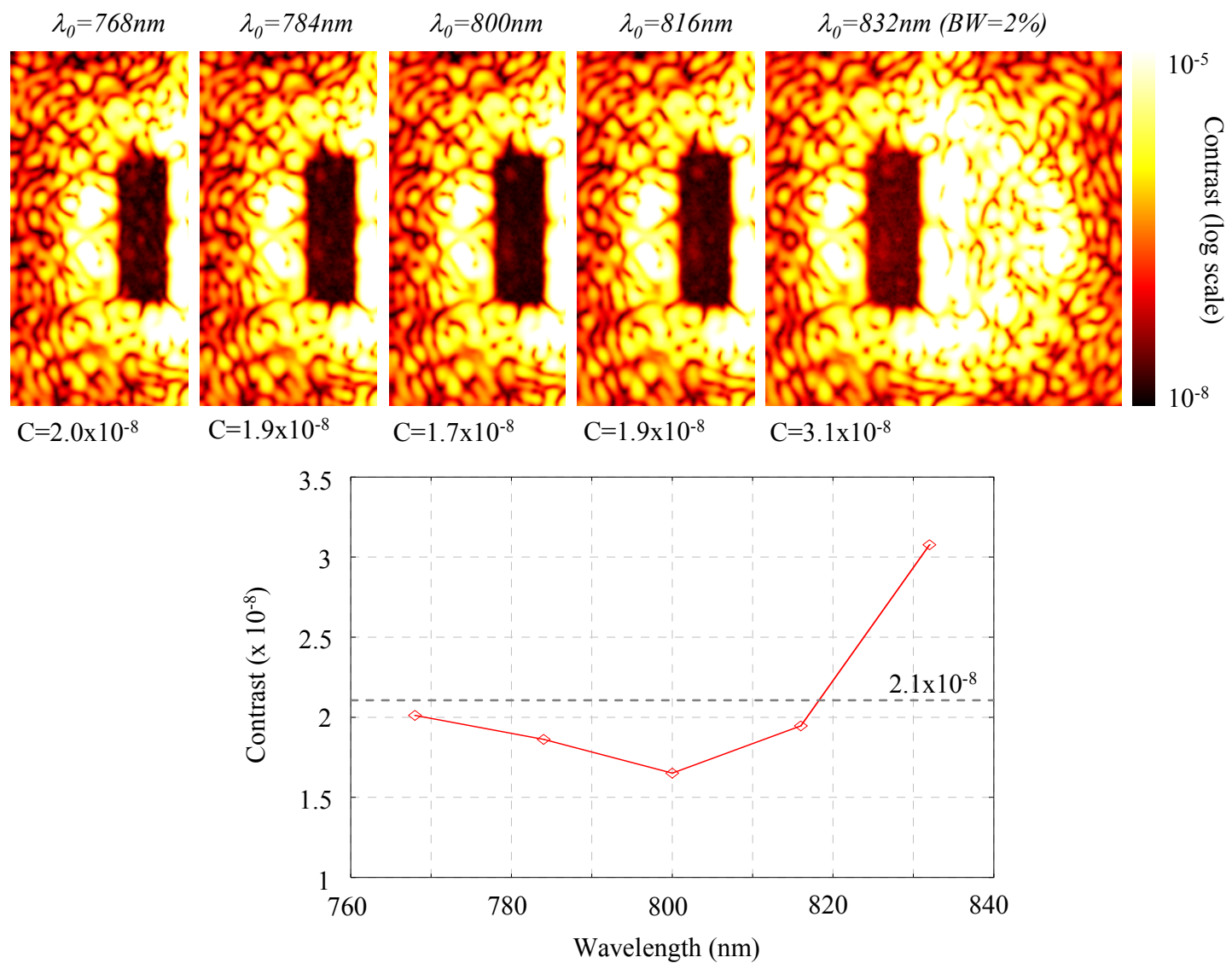

Figure 4 . The $8 \mathrm{OPM}$ coronagraph images acquired with five $2 \%$ bandpass filters. The bottom graph shows the achieved contrasts over the dark holes, as a function of the central wavelengths of the $2 \%$ bandpass filters.

\section{COMBINATION WITH DIFFENTIAL IMAGING TECHNIQUES}

It is expected that combination of the focal-plane phase-mask coronagraphs with differential imaging techniques will enhance the achievable contrast. We built a coronagraph simulator at the laboratory in Hokkaido University, and carried out laboratory demonstration of the angular differential imaging combined with the 8OPM coronagraph, and a polarization differential imaging (or a dual-channel imaging polarimetry) combined with the VVC.

\subsection{An angular differential imaging (ADI)}

A drawback of the 8OPM coronagraph is an on-sky dead zone along the phase transitions of the mask, where planetary light is strongly reduced. However, this drawback can be mitigated by combining the 8OPM coronagraph with the ADI technique ${ }^{24}$. The ADI technique is a powerful tool for reducing a quasi-static speckle noise utilizing a field rotation during tracking of targets by altitude/azimuth telescopes. The field rotation of the ADI observational mode is advantageous for the 8OPM coronagraph, because the planetary image moves across the dead zone as illustrated in an on-sky transmission map in fig. 5a. We note that the ADI will not be efficient at very small angular separation where a potential companion might not move enough to reduce the ADI data. 
We carried out laboratory demonstration of the 8OPM/ADI observational mode. A monochromatic He-Ne laser linked with a single-mode optical fiber is used for simulating a model star. A model planet is numerically added in the experimentally acquired coronagraphic images. A preliminary result of the 8OPM coronagraph without and with the ADI is shown in fig. $5 \mathrm{~b}$ and c, respectively. We set planet/star intensity ratios to $5 \times 10^{-7}$, and star-planet angular separations to $5 \lambda / D$. For the ADI mode, we acquired seven images with the field rotation from $0^{\circ}$ to $36^{\circ}$ with a $6^{\circ}$ step. We can see that the image of the model planet can be clearly detected by the ADI.

(a) On-sky transmission map

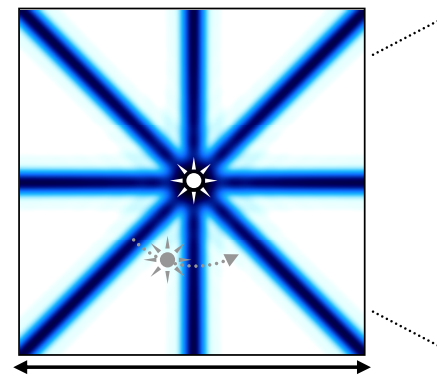

$20 \lambda / \mathrm{D}$ (b) 8 OPM only

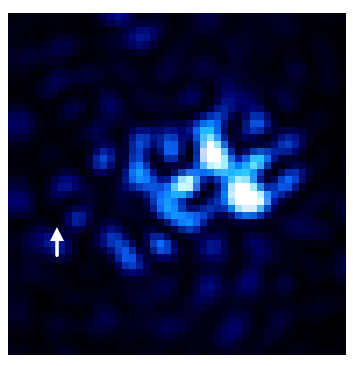

(c) 8 OPM with ADI mode

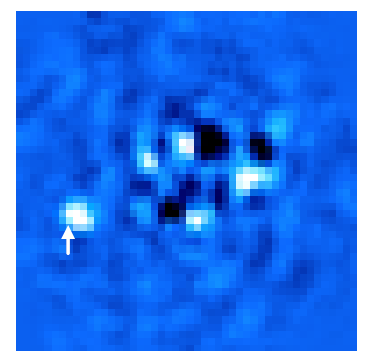

Figure 5. (a) On-sky transmission map of the 8OPM coronagraph. The map has a dead zone along phase transitions. The ADI mode rotates a field-of-view to avoid the dead zone. $(b, c)$ Laboratory demonstration of the 8OPM coronagraph without and with the ADI mode, respectively. After the ADI, a model planetary image (a planet/star ratio of $5 \times 10^{-7}$ ) can be clearly detected at $5 \lambda / D$.

\subsection{A dual-channel imaging polarimetry}

A polarization differential imaging (PDI), or an imaging polarimetry, is also very attractive not only for reducing the residual speckle noise but also for characterizing planetary atmospheres and surfaces through polarization ${ }^{25,26}$. Because the photonic-crystal coronagraph masks use the polarization filtering for achromatization, we can easily introduce the PDI observational mode by putting polarization modulator such as a rotating wave plate in front of the coronagraph optics.

A drawback of the polarization-filtered focal-plane coronagraph masks is loss of intensity due to the polarizing optics. By mitigating the effect of this drawback, a dual-channel coronagraphic configuration, based on polarizing beam splitters (PBS), has been proposed ${ }^{14,27}$. When the dual-channel coronagraph is constructed, efficient high-contrast polarimetric observations can be realized by introducing a polarizing-beam splitter behind the coronagraph to observe coronagraph images of two orthogonally polarized components simultaneously. Figure 6a shows a schematic of the dual-channel coronagraph for the PDI observational mode. The polarization modulator in front of the coronagraph optics is used for reducing non-common-path aberrations due to the dual-channel configuration.

We show a result of our preliminary laboratory demonstration of the dual-channel imaging polarimetry with the photonic-crystal VVC mask. We used He-Ne laser light for simulating unpolarized star light and partially polarized planetary light. Unpolarized starlight and partially polarized planetary light (with a degree of polarization of about $45 \%$ ) were simulated by $45^{\circ}$ and $32^{\circ}$ linearly polarized light, respectively. We roughly set a planet/star intensity ratio to $1 \times 10^{-4}$ and a star-planet angular separation to $5 \lambda / D$.

As can be seen in fig. 6b, two coronagraph images of orthogonally polarized components are observed simultaneously on the same detector. The horizontal $(\mathrm{X})$ and vertical $(\mathrm{Y})$ polarized components can be switched between these two images by the polarization modulator. The modulation of polarized light enables to derive a polarimetric image (Stokes $+\mathrm{Q}$ and $-Q$ images shown in fig. 6c). A difference between the Stokes $+Q$ and $-Q$ images can be calculated to derive the Stokes $+2 \mathrm{Q}$ image for enhancing the planetary light (so-called double difference technique ${ }^{28}$ ). We note that the PDI will be effective for an observed object with significant degrees of polarization. So the gain of the PDI needs to be normalized by the degree of polarization of the object. 

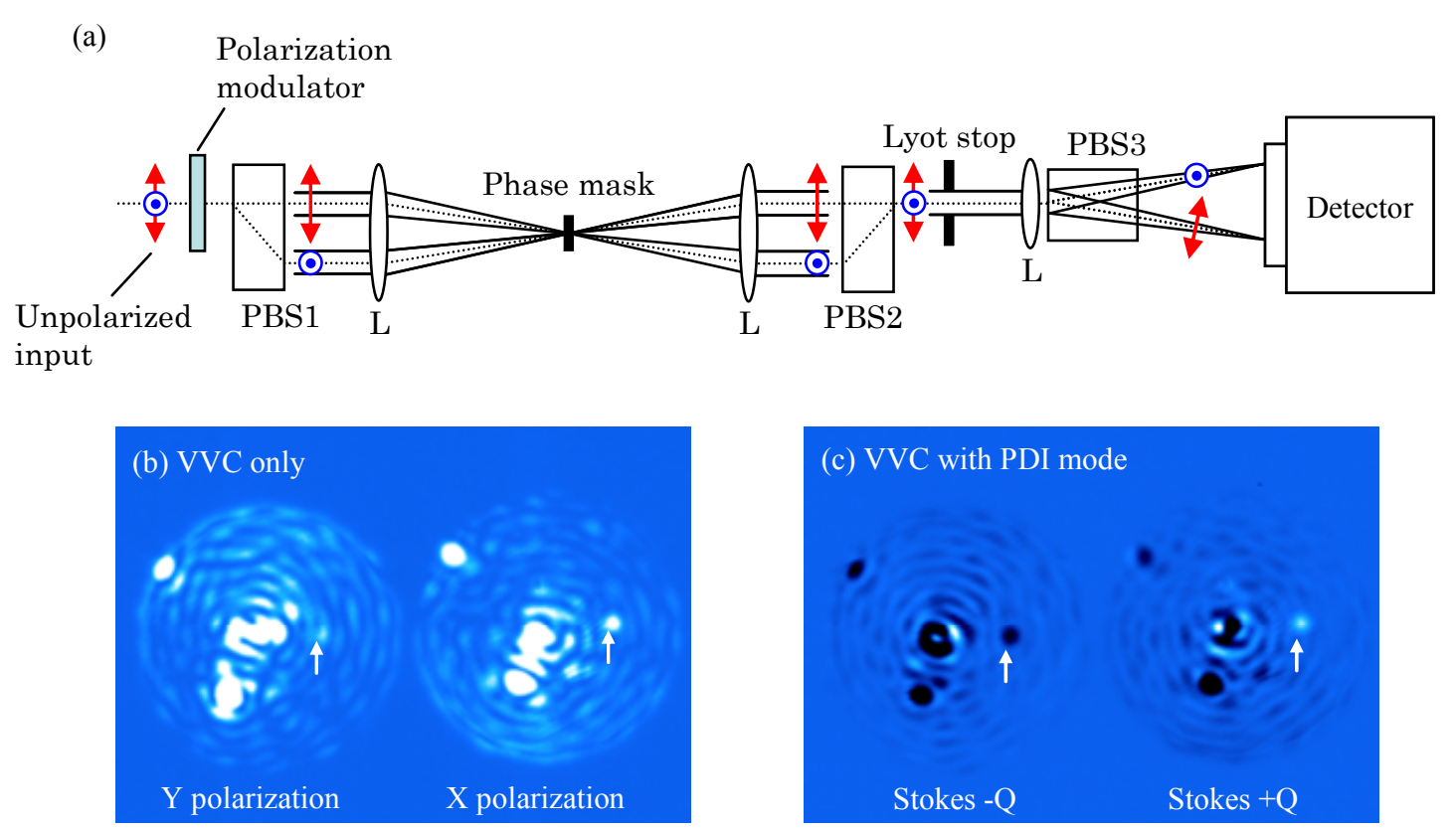

Figure 6. (a) A schematic of a fully-achromatic dual-channel coronagraph for the PDI observational mode. We note that quarter-wave plates (QWPs) are placed behind PBS1 and in front of PBS2 when the VVC mask is used on the focal plane. The QWPs are not needed for the 8OPM. The bottom images show results of the laboratory demonstration of the VVC (b) without and (c) with the polarimetric mode. Planetary images can be detected clearly as positive and negative spots (indicated by arrows), corresponding to the Stokes $+Q$ and $-Q$ images, by the polarimetric mode. An intensity of the partially polarized model planet, located at $5 \lambda / D$, is roughly set to be $1 \times 10^{-4}$ (a degree of polarization of about $45 \%$ ) with respect to that of the model star.

\section{CONCLUSIONS}

We manufactured the photonic crystal focal-plane coronagraph masks, 8OPM and VVC mask, based on the autocloning technique. The manufacturing defects of the photonic crystal masks are extremely small, which have been roughly estimated to be about $400 \mathrm{~nm}$ for a central singular point and about $100 \mathrm{~nm}$ for octant boundaries. The manufactured masks are composed of wave plates with space-variant fast axes, whose retardations are set to $\pi$ (half-wave plate) at designed wavelengths. The masks provide phase modulations as a function of an orientation angle of the fast axes by the Pancharatnam-Berry's phase. Furthermore, the phase modulation can be achromatized by using the appropriate polarization filters.

We carried out laboratory demonstration of the 8OPM coronagraph at the HCIT/JPL, the state-of-the-art coronagraph simulator, in which the Xinetics $64 \times 64 \mathrm{DM}$ is incorporated for the wavefront correction. As a result, we attained average contrast of $2 \times 10^{-8}$ across the five wavelengths over the $10 \%$ bandwidth around $800 \mathrm{~nm}$.

We also proposed the photonic-crystal mask coronagraphs combined with differential imaging techniques to reach higher contrast. First, we proposed to apply an angular-differential imaging (ADI) technique to the 8OPM coronagraph. The ADI mode is advantageous for the 8OPM and the other segment-type phase masks because it mitigates the effect of an intensity loss due to a phase transition of the mask and provides a full field of view around central stars. It is important to remind that the ADI will not be efficient at very small angular separation where a potential companion might not move enough to reduce the ADI data. Next, we also reported a dual-channel imaging polarimetric mode with the VVC. In this observational mode, a two-channel coronagraph based on polarizing beam splitters is built to avoid loss of intensity due to the polarization filters. The polarimetric mode will be effective for reducing unpolarized starlight to detect an object with significant degrees of polarization. We presented the results of the preliminary laboratory demonstrations of the both observational modes. 


\section{ACKNOWLEDGEMENTS}

We acknowledge Takayuki Kawashima of the Photonic Lattice Inc. for providing us with useful information on a photonic-crystal device. This works were carried out at the Hokkaido University, and at the Jet Propulsion Laboratory (JPL), California Institute of Technology, under a contract with the National Aeronautics and Space Administration (NASA). This research was partially supported by the National Astronomical Observatory of Japan (NAOJ), the Japan Aerospace Exploration Agency (JAXA), and the Japan Society for the Promotion of Science (JSPS) through KAKENHI (21340041, 23740139). MT is supported by KAKENHI 22000005.

\section{REFERENCES}

[1] Kalas, P., Graham, J. R., Chiang, E., Fitzgerald, M. P., Clampin, M., Kite, E. S., Stapelfeldt, K., Marois, C. and Krist, J., "Optical images of an exosolar planet 25 light-years from Earth," Science, 322, 1345-1348 (2008).

[2] Marois, C., Macintosh, B., Barman, T., Zuckerman, B., Song, I., Patience, J., Lafrenière, D. and Doyon, R., "Direct imaging of multiple planets orbiting the star HR 8799," Science, 322, 1348-1352 (2008).

[3] Lagrange, A.-M., Bonnefoy, M., Chauvin, G., Apai, D., Ehrenreich, D., Boccaletti, A., Gratadour, D., Rouan, D., Mouillet, D., Lacour, S. and Kasper, M., "A giant planet imaged in the disk of the young star $\beta$ Pictoris," Science, 329, 57-59 (2010).

[4] Rouan, D., Riaud, P., Boccaletti, A., Clénet, Y. and Labeyrie, A., "The four-quadrant phase-mask coronagraph. I. principle," Publ. Astron. Soc. Pacific, 112, 1479-1486 (2000).

[5] Murakami, N., Uemura, R., Baba, N., Nishikawa, J., Tamura, M., Hashimoto, N. and Abe, L., "An eight-octant phase-mask coronagraph," Publ. Astron. Soc. Pacific, 120, 1112-1118 (2008).

[6] Foo, G., Palacios, D. M. and Swartzlander, Jr., G. A., "Optical vortex coronagraph," Opt. Lett., 30, 3308-3310 (2005).

[7] Mawet, D., Riaud, P., Absil, O. and Surdej, J., "Annular groove phase mask coronagraph," Astrophys. J., 633, 1191-1200 (2005).

[8] Carlotti, A., Ricort, G. and Aime, C., "Phase mask coronagraphy using a Mach-Zehnder interferometer," Astron. Astrophys., 504, 663-671 (2009).

[9] Pancharatnam, S., "Generalized theory of interference, and its applications," Proc. Indian Acad. Sci., 44, 247262 (1956).

[10] Berry, M., "The adiabatic phase and Pancharatnam's phase for polarized light," J. Mod. Opt., 34, 1401-1407 (1987).

[11] Kawakami, S., Kawashima, T. and Sato, T, "Mechanism of shape formation of three-dimensional periodic nanostructures by bias sputtering," Appl. Phys. Lett., 74, 463-465 (1999).

[12] Kawashima, T., Miura, K., Sato, T. and Kawakami, S., "Self-healing effects in the fabrication process of photonic crystals," Appl. Phys. Lett., 77, 2613-2615 (2000).

[13] Murakami, N., Nishikawa, J., Yokochi, K., Tamura, M., Baba, N. and Abe, L., "Achromatic eight-octant phasemask coronagraph using photonic crystal," Astrophys. J., 714, 772-777 (2010).

[14] Baba, N., Murakami, N., Ishigaki, T. and Hashimoto, N., "Polarization interferometric stellar coronagraph," Opt. Lett., 27, 1373-1375 (2002).

[15] Mawet, D., Serabyn, E., Liewer, K., Hanot, C., McEldowney, S., Shemo, D. and O'Brien, N., "Optical vectorial vortex coronagraphs using liquid crystal polymers: theory, manufacturing and laboratory demonstration," Opt. Express 17, 1902-1918 (2009).

[16] Trauger, J. T. and Traub, W. A. "A laboratory demonstration of the capability to image an Earth-like extrasolar planet," Nature, 446, 771-773 (2007).

[17] Trauger, J., Moody, D., Gordon, B., Krist, J. and Mawet, D., "A hybrid Lyot coronagraph for the direct imaging and spectroscopy of exoplanet systems: recent results and prospects," Proc. SPIE, 8151, 81510G (2011).

[18] Belikov, R., Give'on, A., Kern, B., Cady, E., Carr, M., Shaklan, S., Balasubramanian, K., White, V., Echternach, P., Dickie, M., Trauger, J., Kuhnert, A. and Kasdin, N. J., "Demonstration of high contrast in 10\% broadband light with the shaped pupil coronagraph," Proc. SPIE, 6693, 66930Y (2007).

[19]Kern, B., Guyon, O., Give'on, A., Kuhnert, A. and Niessner, A., "Laboratory testing of a Phase-Induced Amplitude Apodization (PIAA) coronagraph," Proc. SPIE, 8151, 815104 (2011). 
[20] Mawet, D, Murakami, N., Delacroix, C., Serabyn, E., Absil, O., Baba, N., Baudrand, J., Boccaletti, A., Burruss, R., Chipman, R., Forsberg, P., Habraken, S., Hamaguchi, S., Hanot, C., Ise, A., Karlsson, M., Kern, B., Krist, J., Kuhnert, A., Levine, M., Liewer, K., McClain, S., McEldowney, S., Mennesson, B., Moody, D., Murakami, H., Niessner, A., Nishikawa, J., O’Brien, N., Oka, K., Park, P., Piron, P., Pueyo, L., Riaud, P., Sakamoto, M., Tamura, M., Trauger, J., Shemo, D., Surdej, J., Tabirian, N., Traub, W., Wallace, J. and Yokochi, K., "Taking the vector vortex coronagraph to the next level for ground- and space-based exoplanet imaging instruments: review of technology developments in the USA, Japan, and Europe," Proc. SPIE, 8151, 815108 (2011).

[21] Serabyn, E., Mawet, D., Wallace, J. K., Liewer, K., Trauger, J., Moody, D. and Kern, B., "Recent progress in vector vortex coronagraphy," Proc SPIE, 8146, 81460L (2011).

[22] Mawet, D., Serabyn, E., Moody, D., Kern, B., Niessner, A., Kuhnert, A., Shemo, D., Chipman, R., McClain, S. and Trauger, J., "Recent results of the second generation of vector vortex coronagraphs on the high-contrast imaging testbed at JPL," Proc. SPIE, 8151, 81511D (2011).

[23] Give'on, A., Kern, B., Shaklan, S., Moody, D. C. and Pueyo, L., "Broadband wavefront correction algorithm for high-contrast imaging systems," Proc. SPIE, 6691, 66910A (2007).

[24] Marois, C., Lafrenière, D., Doyon, R., Macintosh, B. and Nadeau, D., "Angular differential imaging: a powerful high-contrast imaging technique," Astrophys. J., 641, 556-564. (2006).

[25] Kuhn, J. R., Potter, D. and Parise, B., "Imaging polarimetric observations of a new circumstellar disk system," Astrophys. J., 553, L189-L191 (2001).

[26] Baba, N. and Murakami, N., "A method to image extrasolar planets with polarized light," Publ. Astron. Soc. Pacific, 115, 1363-1366 (2003).

[27] Murakami, N., Inabe, T., Komatsu, T., Nishikawa, J., Hashimoto, N., Kurihara, M., Baba, N. and Tamura, M., "Polarization-interferometric eight-octant phase-mask coronagraph using ferroelectric liquid crystal for exoplanet detection," Proc. SPIE, 7731, 77314E (2010).

[28] Hinkley, S., Oppenheimer, B. R., Soummer, R., Brenner, D., Graham, J. R., Perrin, M. D., Sivaramakrishnan, A., Lloyd, J. P., Roberts, Jr., L. C., and Kuhn, J., "Speckle suppression through dual imaging polarimetry, and a ground-based image of the HR 4796A circumstellar disk," Astrophys. J., 701, 804-810 (2009). 